Research Article

\title{
Existence of Limit Cycles for a Class of Quartic Polynomial Differential System Depending on Parameters
}

\author{
Sarah Abdullah Qadha $\mathbb{D}^{1},{ }^{1,2}$ Muneera Abdullah Qadha ${ }^{(D)}{ }^{1,2}$ and Haibo Chen ${ }^{1}$ \\ ${ }^{1}$ School of Mathematics and Statistics, Central South University, Changsha 410083, China \\ ${ }^{2}$ Department of Mathematics, Faculty of Education at Al-Mahweet, Sana'a University, Al-Mahweet, Yemen
}

Correspondence should be addressed to Sarah Abdullah Qadha; sarah_qadha@csu.edu.cn

Received 20 April 2021; Revised 6 July 2021; Accepted 12 July 2021; Published 27 July 2021

Academic Editor: Firdous A. Shah

Copyright ( $) 2021$ Sarah Abdullah Qadha et al. This is an open access article distributed under the Creative Commons Attribution License, which permits unrestricted use, distribution, and reproduction in any medium, provided the original work is properly cited.

We studied the existence of limit cycles for the quartic polynomial differential systems depending on parameters. To prove that, first, we used the formal series method based on Poincare' ideas to determine the center-focus. Then, by the Hopf bifurcation theory, we obtained the sufficient condition for the existence of the limit cycles. Finally, we provided some numerical examples for illustration.

\section{Introduction}

In the twentieth century, progress in applied electronics has been rapid. Physicists invented the triode vacuum tube, making stable self-excited oscillations of constant amplitude. Thus, propagating sound and pictures through electronics became possible. However, it was not possible to describe this oscillation phenomenon by linear differential equations. In [1] 1926, van der Pol first obtained a differential equation to describe oscillations of the constant amplitude of a triode vacuum tube, as follows:

$$
\ddot{x}+\mu\left(x^{2}-1\right) \dot{x}+x=0 .
$$

This was the main reason for the appearance of the nonlinear equations and qualitative theory. One of the main issues in analysing the qualitative theory of differential equations is the limit cycle. The limit cycle was discovered by Poincare' (1881-1886). Recently, the problem of limit cycles has become increasingly critical and has attracted the attention of many pure and applied mathematicians, see for instance [1-10]. The necessary problems in the qualitative theory of differential equation include the existence of the limit cycle.
Existence, nonexistence, uniqueness, and other properties of limit cycles have been studied extensively by mathematicians and physicists. Then, since the 1950s, many mathematical models in physics, engineering, chemistry, biology, and economics have been displayed as autonomous plane systems with limit cycles. Many researchers have studied the existence of limit cycles for the quadratic and cubic systems, and numerous research achievements have been obtained, see for instance [3, 5, 7, 10-18].

For examples, consider the following system:

$$
\begin{aligned}
& \frac{\mathrm{d} x}{\mathrm{~d} t}=P(x, y), \\
& \frac{\mathrm{d} y}{\mathrm{~d} t}=Q(x, y),
\end{aligned}
$$

where $P(x, y)$ and $Q(x, y)$ denote homogeneous polynomial of degree $n$ and real variable $x, y$ and some parameter.

Consider the following system:

$$
\begin{aligned}
& \frac{\mathrm{d} x}{\mathrm{~d} t}=-y+P_{n}(x, y), \\
& \frac{\mathrm{d} y}{\mathrm{~d} t}=x+Q_{n}(x, y),
\end{aligned}
$$


where $P_{n}(x, y)$ and $Q_{n}(x, y)$ are polynomials of degree $n$, with real coefficients without constant terms. System (3) always has either a center or fine (weak) focus at the origin. Distinguishing between the center and a focus at the origin is the main problem.

We know that linear differential system $(\mathrm{d} x / \mathrm{d} t)=-y,(\mathrm{dy} / \mathrm{d} t)=x$ can have the center. However, the perturbation of this center inside the class of linear differential systems cannot produce a limit cycle because the linear differential system cannot have an isolated periodic solution in the set of all periodic solutions:

(1) When $n=2$, system (3) is the quadratic polynomial differential system whose centers have been completely classified. Many papers study how many limit cycles can bifurcate from the periodic orbits of these centers. As an example, consider the following system:

$$
\begin{aligned}
\frac{\mathrm{d} x}{\mathrm{~d} t}= & -y(1+x+\alpha y), \\
\frac{\mathrm{d} y}{\mathrm{~d} t}= & x+(\lambda+\beta+\gamma) y+a x^{2} \\
& +(\alpha+\beta+\gamma) x y+c \gamma y^{2} .
\end{aligned}
$$

In [19], the authors obtained a quadratic system that has at most four limit cycles and only in the $(3: 1)$ distribution when $a=(1 / 2), c=-1$.

(2) When $n=3$, system (3) is a cubic system.

In [20], Malkin obtained the necessary and sufficient conditions for system (3) for the origin to be the center. In [21], the authors proved that these conditions were satisfactory for the integrability of the system. In [22], Liu derived the formulas of the first five focus values of the origin for system (3) and proved that the origin is a center if the five focus values are all zero. This finding implies that a central symmetric cubic system with respect to the origin has at most five small amplitude limit cycles around the origin (see [23]).

Consider the following system:

$$
\begin{aligned}
& \frac{\mathrm{d} x}{\mathrm{~d} t}=-y(1-a x)+(1-b x)+a_{1} x+a_{2} x^{2}+a_{3} x^{3}, \\
& \frac{\mathrm{d} y}{\mathrm{~d} t}=x(1-c x)(1-d x) .
\end{aligned}
$$

In [24], Quan obtained the sufficient and necessary condition for the existence of a limit cycle in the case $a_{2}=0, a=c$, and $b=d$. The authors of [25] studied the condition for the existence of limit cycles in the system when $a=0$ and $b=d$. One article [26] showed that the case $a=$ $c$ and $b=d=0$ has no limit cycle.

In this paper, we will study the existence of limit cycles for the following quartic polynomial differential systems depending on parameters:

$$
\begin{aligned}
& \frac{\mathrm{d} x}{\mathrm{~d} t}=-y+k x^{2}+l x y, \\
& \frac{\mathrm{dy}}{\mathrm{d} t}=x+m x^{2} y+n y^{4},
\end{aligned}
$$

where $k, l, m$, and $n$ are parameters, in which the linear system is the center. If the singular point for nonlinear is the center, quartic system (6) does not have a limit cycle. If the singular point for nonlinear systems is the focus or node, then quartic system (6) has the limit cycle. System (6) only has one singular point on the origin. It does not have any singular point on the neighborhood; so, the origin (the singular point) is isolated.

In the first section of this paper, we presented some necessary definitions and theorem. In the second section, we used a formal series method based on Poincare' ideas to determine the center-focus. By using the Hopf bifurcation theory, we obtained the sufficient condition for the existence of limit cycle for quartic system (6), which bifurcated from the equilibrium point (singular point). We also obtained sufficient conditions for the existence of the limit cycle according to the change analysis of the stability of the focus when the parameters change. Finally, we provided some examples for illustration.

\section{Some Preliminary Results}

In this section, we introduce some definitions and notations that we will need for the existence of the quartic differential system (6).

Theorem 1 (see [1]). Closed trajectories of different vector fields of a complete family do not intersect.

Theorem 2 (Bendixson's criterion) (see [27]). If $P_{x}, Q_{y}$ is continuous in $R$ which is simply connected and $\operatorname{div} \vec{F}=(\partial P / \partial x)+(\partial Q / \partial y) \neq 0$ at any point of $R$, then the system has no closed trajectories inside $R$.

Definition 1 (Hopf bifurcation of order $k$ ) (see [28], page 385). Consider the following system:

$$
\begin{aligned}
& \frac{\mathrm{d} x}{\mathrm{~d} t}=P(x, y, \eta), \\
& \frac{\mathrm{d} y}{\mathrm{~d} t}=Q(x, y, \eta),
\end{aligned}
$$

where $x, y \in \mathbb{R}^{1}, \eta \in \mathbb{R}^{n}$, and $P, Q \in C^{\infty}$, when two eigenvalues $\lambda_{1,2}=a(\eta) \pm i b(\eta)$ of the linear part of the system above (7) cross the imaginary axis. We say that system (7) has a Hopf bifurcation of order $k(k>1)$ at the origin if

(1) $a(0)=0$

(2) $b(0)=b_{0} \neq 0$

(3)

$$
\operatorname{Re}\left(C_{1}\right)=\operatorname{Re}\left(C_{2}\right)=\cdots=\operatorname{Re}\left(C_{k-1}\right)=0, \quad \operatorname{Re}\left(C_{k}\right) \neq 0
$$

where $C_{1}, \ldots, C_{k}$ are the coefficients of Poincaré-Lyapunov constants.

In this case, we also say that the origin is a weak focus of order $k$.

Note: In two dimensions, when the focus Point switches from stable to unstable (or vice versa), then a periodic solution appears and a Hopf bifurcation occurs. [29]. 
Theorem 3 (see [28], page 385). If we let system (2) be a $C^{\infty}$ with an equilibrium $(0,0)$, that is, a weak focus of order $k$, then

(1) If $n \geq k$, and (5) $\eta_{\eta=0}=(2)$, then there is $\delta>0$ and $a$ neighborhood $\Gamma$ of $(x, y)=(0,0)$ exists. $|\eta|<\delta$, (5) has at most $k$ limit cycles in neighborhood $\Gamma$

(2) For any integer $i, 1 \leq i \leq k$ and a neighborhood $\Gamma^{*} \subset \Gamma$ of $(x, y)=(0,0)$, there exists a system of the form (5) $\eta_{\eta=0}$ with $(5)_{\eta=0}=(2)$, and a number $\delta^{*}>0$, such that $(5)_{\eta=0}$ has exactly $i$ limit cycles in $\Gamma^{*}$ for $\eta \in S$, where $S$ is an open subset of $\left(\eta / 0<|\eta|<\delta^{*}\right)$ and $0 \in \bar{S}$

Remark 1 (see [27]). The linear part of system (6) has closed trajectories because the linear system has $\operatorname{Tr} A=P_{x}(A)+$ $Q_{y}(A)=0$ and $\operatorname{det} A=P_{x}(A) Q_{y}(A)-P_{y}(A) \quad Q_{x}(A)=$ $1>0$ where $A$ is a singular point for system. $\lambda_{1}$ and $\lambda_{2}$ are two conjugate pure imaginary roots. Then, the linear system (6) can be transformed into the following:

$$
\begin{aligned}
& r \dot{r}=x \dot{x}+y \dot{y}, \quad \text { then } \dot{r}=0, \\
& r^{2} \dot{\theta}=x \dot{y}-y \dot{x}, \quad \text { then } \dot{\theta}=1,
\end{aligned}
$$

whose solution is as follows:

$$
\begin{aligned}
& r=r_{0} \geq 0, \\
& \theta=t+\theta_{0} .
\end{aligned}
$$

The orbit form has a family of closed curves around the origin A. The singular point is called a center. The simulation is presented in Figure 1.

\section{Main Results}

\subsection{Determine the Center-Focus and It Is Stable}

Theorem 4. For system (6), we have the following:

(1) If $(2 k l-m) \neq 0, k l \neq 0,((m+k l) / 4)<0$, then $A(0,0)$ is an unstable first-order weak focus

(2) If $(2 k l-m) \neq 0, k l \neq 0,((m+k l) / 4)>0$, then $A(0,0)$ is a stable first-order weak focus

(3) If $(2 k l-m)=0, k l=0$, then the point $A(0,0)$ is the center point

(4) If $k l=0,(2 k l-m)>0$, then the point $A(0,0)$ is an unstable second-order weak focus

(5) If $k l=0 \quad(2 k l-m)<0$, then the point $A(0,0)$ is a stable second-order weak focus

(6) If $(2 k l-m)=0, k l<0$, then the point $A(0,0)$ is an unstable third-order weak focus

(7) If $(2 k l-m)=0, k l>0$, then the point $A(0,0)$ is a stable third-order weak focus

Proof. By using the formal series method based on Poincare' ideas, we can easily obtain these results of this theorem. The details are omitted here.

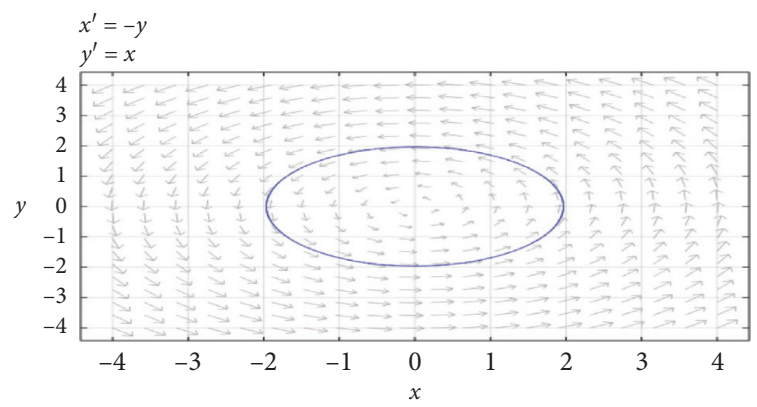

Figure 1: Center.

\subsection{The Nonexistence of Limit Cycle}

Theorem 5. If $(2 k l-m)=0, k l=0$, then system (6) has no limit cycle in the whole plane.

Proof. When the conditions above hold, then the point $A(0,0)$ is the center point. And, when $k l=0, m=0, \quad$ then $\quad P(-x, y)=P(x, y), Q(-x, y)=$ $-Q(x, y)$. The vector field $(P(x, y), Q(x, y))$ is symmetrical with respect to $y$-axis. So, there is no limit cycle in the neighborhood of point $A(0,0)$ when the conditions of Theorem 5 hold. The simulation is presented in Figure 2.

\subsection{Existence of Limit Cycles}

Theorem 6. When one of the following conditions holds, system (6) has at least one limit cycle around $A(0,0)$ :

(1) $((k l+m) / 4)<0, k l \neq 0,(2 k l-m) \neq 0,-1 \ll m<0$

(2) $((k l+m) / 4)>0, k l \neq 0,(2 k l-m) \neq 0,0<m \ll 1$

(3) $(2 k l-m)>0, k l=0,-1 \ll m<0$

(4) $(2 k l-m)<0, k l=0,0<m \ll 1$

(5) $(m-2 k l)=0, k l<0,-1 \ll m<0$

(6) $(m-2 k l)=0, k l>0,0<m \ll 1$

Proof. For conditions (1), (3), and (5), the singular point $A(0,0)$ is an unstable weak focus point of system (6). When $m$ decreases from zero, the singular point $A(0,0)$ of system (6) changes from an unstable weak focus to a stable weak focus. According to the Hopf bifurcation theory, these parameters change because when the stability changes, system (6) generates at least one unstable limit cycle in the neighborhood of $A(0,0)$.

For condition (2), (4), and (6), the singular point $A(0,0)$ is the stable weak focus point of system (6). When $m$ increases from zero, the singular point $A(0,0)$ of system (6) changes from a stable weak focus to an unstable weak focus, and according to the Hopf bifurcation theory, under these parameters, system (6) generates at least one stable limit cycle in the neighborhood of $A(0,0)$.

Theorem 7. System (6) has at least three limit cycles bifurcating from the origin $A(0,0)$ 


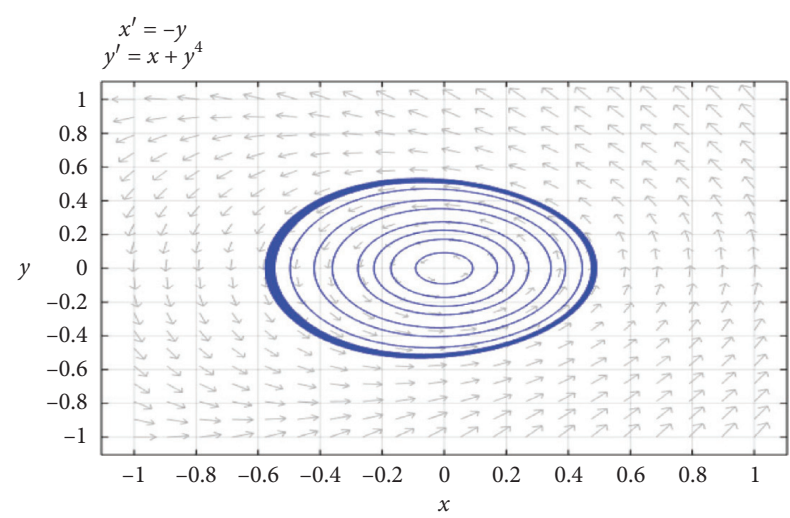

Figure 2: Nonexistence of limit cycle for system (6).

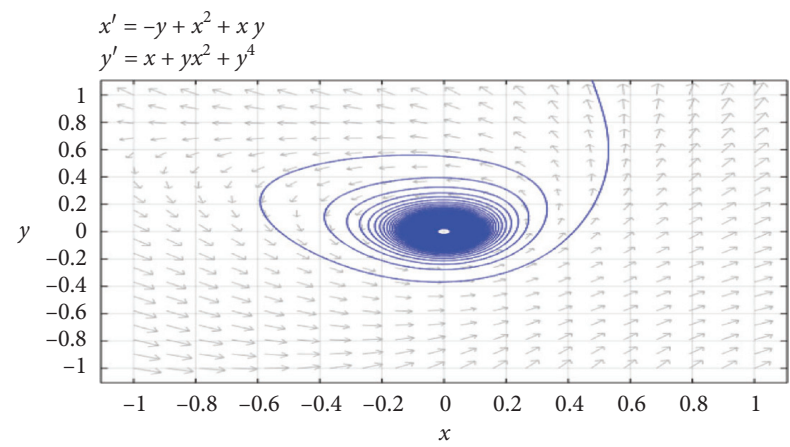

Figure 3: Existence of limit cycle for system (6), when $k=1, l=1$, $m=1, n=1$.

Proof. From Theorem 4, we determine that system (6) has three weak focuses. We denote the number of weak focus as $k$, and $k=3$. System (6) is a class quartic; thus, we denote $n$ as $n=4$ According to Theorem 3 , we determine that system (6) has three limit cycles around $A(0,0)$

\subsection{Numerical Solution}

Example 1. In system (6), if we take the values of the parameters as $\mathrm{k}=1, \mathrm{l}=1, \mathrm{~m}=1$, and $\mathrm{n}=1$, then a limit cycle exists. The simulation is presented in Figure 3.

Example 2 (the existence). In system (6), if we take values of the parameters as $k=0.2, l=-0.2, m=-0.06$, and $n=1$, then by Theorem 6 Case 1 , there exists a limit cycle around $A(0,0)$. The simulation is presented in Figure 4 .

In system (6), if we take values of the parameters as $k=-1, l=1, m=1$, and $n=1$, then by Theorem 6 Case 2, there exists a limit cycle around $A(0,0)$. The simulation is presented in Figure 5.

In system (6), if we take values of the parameters as $k=1, l=0, m=-0.2$, and $n=1$, then by Theorem 6 Case 3 , there exists a limit cycle around $A(0,0)$. The simulation is presented in Figure 6.

In system (6), if we take values of the parameters as $k=-1, l=0, m=1$, and $n=1$, then by Theorem 6 Case 4 ,

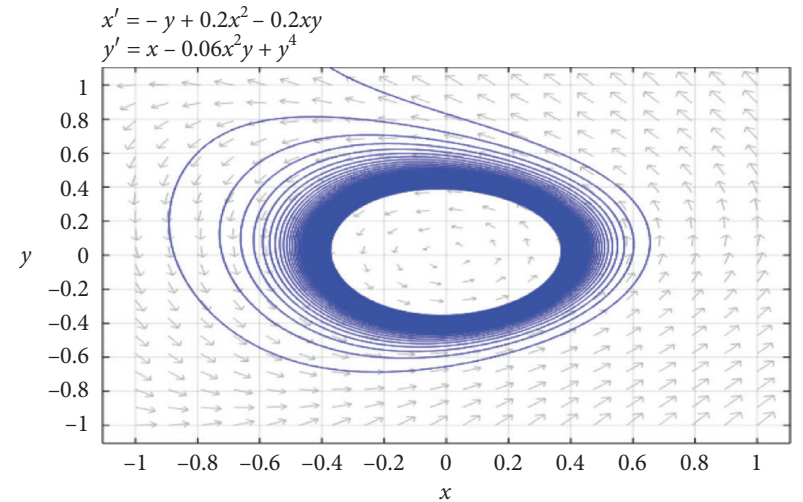

Figure 4: Existence of limit cycle of system (6), when $k=0.2, l=-0.2, m=-0.06$, and $n=1$.

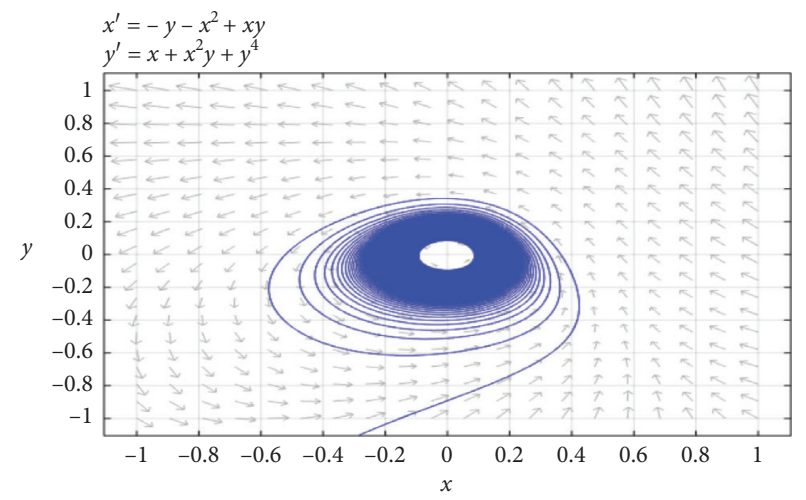

Figure 5: Existence of limit cycle of system (6), when $k=-1, l=1, m=1$, and $n=1$.

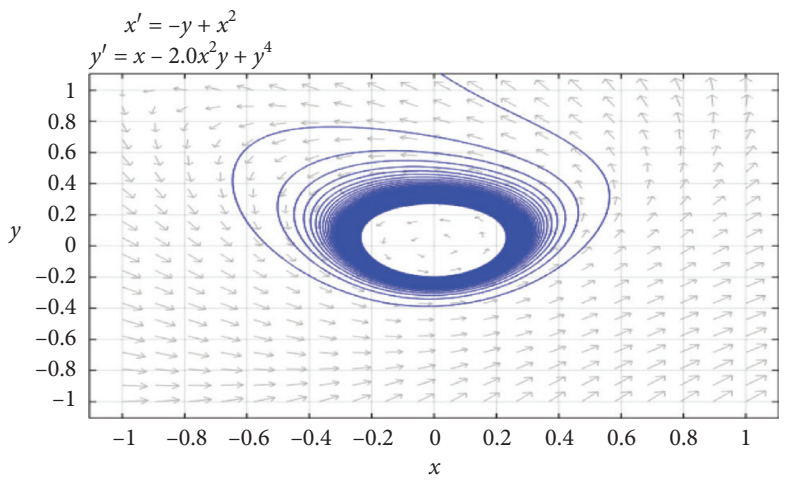

Figure 6: Existence of limit cycle of system (6), when $k=1, l=0, m=-0.2$, and $n=1$.

there exists a limit cycle around $A(0,0)$. The simulation is presented in Figure 7.

In system (6), if we take values of the parameters as $k=0.25, l=-0.25, m=-0.125$ and $n=1$, then by Theorem 6 Case 5 , there exists a limit cycle around $A(0,0)$. The simulation is presented in Figure 8.

In system (6), if we take values of parameters as $k=-0.25, l=-0.25, m=0.125$, and $n=1$, then by Theorem 6 Case 6 , there exists a limit cycle around $A(0,0)$. The simulation is presented in Figure 9. 


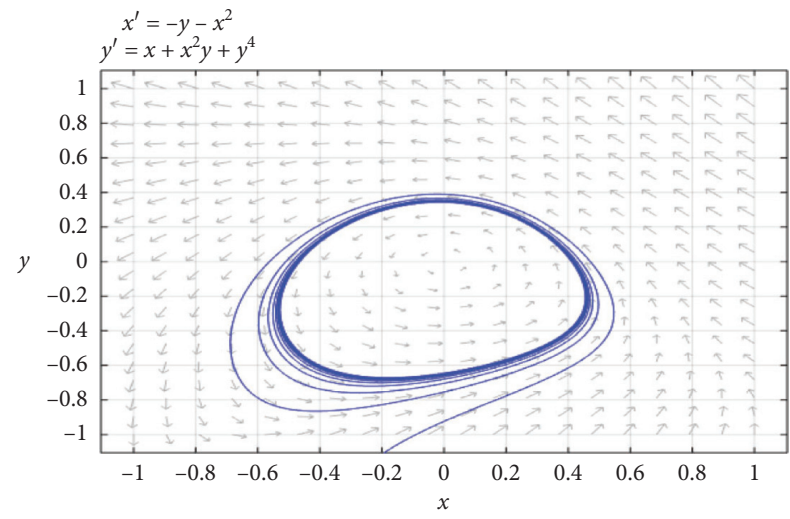

Figure 7: Existence of limit cycle of system (6), when $k=-1, l=0, m=1$, and $n=1$.

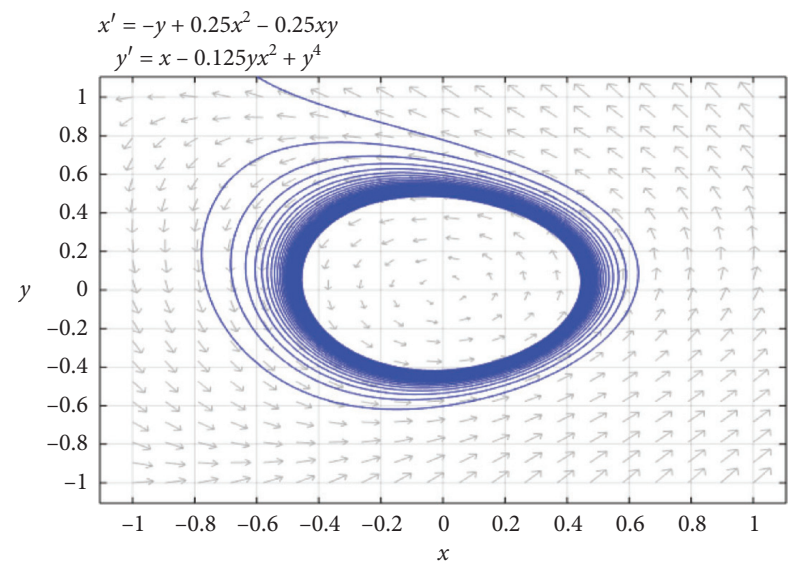

Figure 8: Existence of limit cycle of system (6), when $k=0.25, l=-0.25, m=-0.125$, and $n=1$.

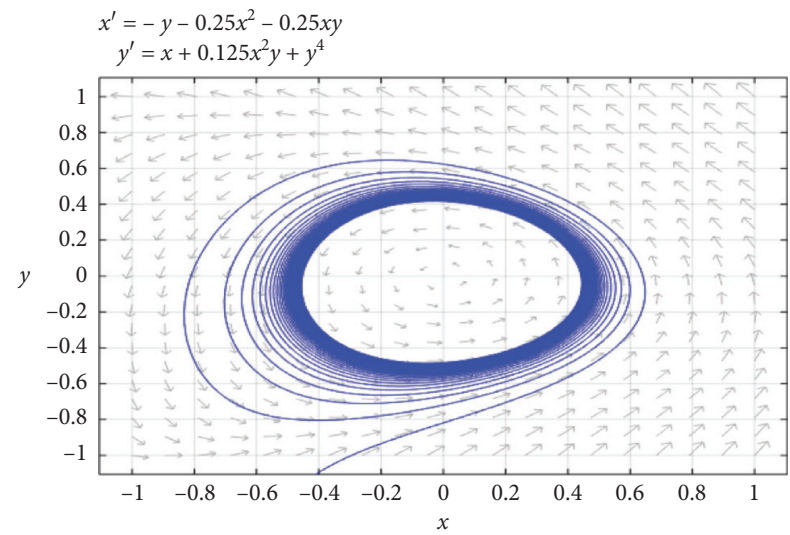

Figure 9: Existence of limit cycle of system (6), when $k=-0.25, l=-0.25, m=0.125$, and $n=1$.

\section{Conclusions}

In this paper, we studied the existence of the limit cycle and used the formal series method to determine the center-focus point. By the Hopf bifurcation theory, we obtained the sufficient condition for the existence of the limit cycles for this system, which bifurcate from the equilibrium point. We provided some examples for illustration.

\section{Data Availability}

All data required for this paper are included within the article.

\section{Conflicts of Interest}

The authors declare that they have no conflicts of interest.

\section{References}

[1] Y.-Q. Ye, "Theory of limit cycles," The American Mathematical Society, vol. 66, 1986.

[2] X. Li, "Limit cycles in a quartic system with a third-order nilpotent singular point," Advances in Difference Equations, vol. 2018, 2018.

[3] C. Bianca and L. Guerrini, "Existence of limit cycles in the Solow model with delayed-logistic population growth," The Scientific World Journal, vol. 2014, Article ID 207806, 8 pages, 2014.

[4] S. P' Erez-Gonź Alez, J. Torregrosa, and P. J. Torres, "Existence and uniqueness of limit cycles for generalized $\phi$-Laplacian Liénard equations," Journal of Chemical Information and Modeling, vol. 53, no. 9, pp. 1689-1699, 2013.

[5] X. G. Liu, B. Liu, and Y. Lv, "Existence and uniqueness and stability of limit cycle for a class of planar nonlinear systems," in Proceedings of the 32nd Chinese Control Conference, Xi'an, China, July 2013.

[6] J. Giné, "Limit cycle bifurcations from a non-degenerate center," Applied Mathematics and Computation, vol. 218, no. 9, pp. 4703-4709, 2012.

[7] K. I. T. Al-Dosary, "Existence and uniqueness of limit cycles in a class of planar differential systems," Journal of Applied Mathematics, vol. 2011, Article ID 497164, 7 pages, 2011.

[8] V. A. Gaiko, "Global bifurcations of limit cycles in the Kukles cubic system,” 2016, http://arxiv.org/abs/1611.08113.

[9] C. Christopher and C. Li, Limit Cycles of Differential Equations, Springer Science \& Business Media, Berlin, Germany, 2007.

[10] D. Xiao and H. Zhu, "Multiple focus and Hopf bifurcations in a predator-prey system with nonmonotonic functional response," SIAM Journal of Applied Mathematics, vol. 66, no. 3, pp. 802-819, 2006.

[11] M. Han, Y. Lin, and P. Yu, "A study on the existence of limit cycles of a planar system with third-degree polynomials," International Journal of Bifurcation and Chaos, vol. 14, no. 1, pp. 41-60, 2004.

[12] B. Coll, A. Gasull, and J. Llibre, "Some theorems on the existence, uniqueness, and nonexistence of limit cycles for quadratic systems," Journal of Differential Equations, vol. 67, no. 3, pp. 372-399, 1987.

[13] T. Liu, F. Li, Y. Liu, and S. Li, "Center-focus determination and limit cycles bifurcation for \$p: $\mathbf{\$}$ homogeneous weight singular point," 2016, http://arxiv.org/abs/1608.08890.

[14] J. Giné, "Non-existence of limit cycles for planar vector fields," Electronic Journal of Differential Equations, vol. 2014, no. 75, 8 pages, 2014.

[15] M. Hayashi, "Unique existence and nonexistence of limit cycles for a classical Liénard system 1 introduction 2 main results and proofs," Advances in Dynamical Systems and Applications, vol. 11, no. 1, pp. 59-65, 2016. 
[16] J. Sugie, A. Kono, and A. Yamaguchi, "Existence of limit cycles for Liénard-type systems with p-Laplacian," Nonlinear Differential Equations and Applications NoDEA, vol. 14, no. 1-2, pp. 91-110, 2007.

[17] D. Xiao and Z. Zhang, "On the existence and uniqueness of limit cycles for generalized Liénard systems," Journal of Mathematical Analysis and Applications, vol. 343, no. 1, pp. 299-309, 2008.

[18] D. Xiao and Z. Zhang, "On the uniqueness and nonexistence of limit cycles for predator prey systems," Nonlinearity, vol. 16, no. 3, pp. 1185-1201, 2003.

[19] V. A. Gaiko and V. A. Gaiko, "Field rotation parameters and limit cycle bifurcations field rotation parameters and limit cycle bifurcations," The Institut des Hautes Études Scientifiques, vol. 9, pp. 2150-2157, 2006.

[20] K. E. Malkin, "Criteria for center of a differential equation," Volg. Matem. Sbornik, vol. 2, pp. 87-91, 1964.

[21] V. A. Lunkevich and K. S. Sibirsky, "Conditions of a center in homogeneous non-linearities of third degree," Differential Equations, vol. 1, pp. 1164-1168, 1965.

[22] Y. Liu, "Formula of focal value, condition of center and central for a class of planar cubic system," Chinese Science Bulletin, vol. 2, no. 85-87, 1987.

[23] R. Benterki and J. Llibre, "Centers and limit cycles of polynomial differential systems of degree 4 via averaging theory," Journal of Computational and Applied Mathematics, vol. 313, pp. 273-283, 2017.

[24] L. J. Quan, "The existence and uniqueness of limit cycles for a class of cubic system," Journal of Systems Science and Mathematical Sciences, vol. 19, no. 1, pp. 16-18, 1999.

[25] A. Algaba and M. Reyes, "Computing center conditions for vector fields with constant angular speed," Journal of Computational and Applied Mathematics, vol. 154, no. 1, pp. 143-159, 2003.

[26] F. C. Hong, "The existence and uniqueness of limit cycles for class of cubic systems," Journal of Ningde Teachers College (Natural Science), vol. 23, no. 2, 2011.

[27] A. Mattuck, "LC. limit Cycles," Journal of Differential Equations, pp. 1-6, 2011.

[28] S.-N. Chow, C. Li, and D. Wang, Normal Forms and Bifurcation of Planar Vector Fields, Cambridge University Press, Cambridge, UK, 1994.

[29] C. Börgers, "Hopf bifurcations," An Introduction to Modeling Neuronal Dynamics, vol. 66, pp. 91-97, 2017. 